\title{
LA COMMUNICATION SANTE : NOUVEL ENJEU DE LA CONSOMMATION ALIMENTAIRE Contexte réglementaire et statut juridique des aliments santé
}

\author{
Health communication: the new stakes in food consumption The legal context and status of health \\ foods
}

Oléagineux, Corps Gras, Lipides. Volume 8, Numéro 4, 336-41, Juillet - Août 2001, Dossier : "Aliments fonctionnels et lipides"

Auteur(s) : Nicole COUTRELIS, Patricia JUCHET, Coutrelis \& Associés - Paris, Bruxelles, 55, avenue Marceau, 75116 Paris, France..

Résumé : À la frontière de l'aliment et du médicament, les " aliments santé " sont aujourd'hui au centre de toutes les attentions - et de toutes les interrogations - du secteur de l'agroalimentaire. La notion n'étant pas définie par la loi et le vocabulaire utilisé variant d'un ouvrage ou d'un auteur à l'autre, la matière est difficilement et diversement appréhendée par les professionnels et les consommateurs. Aussi, afin d'assurer à la présente étude la cohérence nécessaire à la compréhension du sujet, lui-même fort complexe, on privilégiera une définition large (et nécessairement subjective) de la notion d'aliments santé 1 . Dès lors, celle-ci recouvrira : - les aliments traditionnels comportant des allégations faisant un lien, quel qu'il soit, avec la santé ; - les aliments dits " fonctionnels"; - les aliments traditionnels supplémentés ; - l'alimentation particulière ; - les compléments alimentaires. Tels que définis, les aliments santé sont soumis à un régime juridique au centre d'un prisme composé de nombreuses réglementations, communautaires et françaises, relatives aux denrées alimentaires et aux médicaments. La multiplicité de ces réglementations ne va pas sans difficulté, leur mise en œuvre manquant parfois de la cohérence qui serait nécessaire à la sécurité juridique. Essentiellement centrée sur l'information et la protection du consommateur, la réglementation actuellement applicable en France est à la fois générale et spécifique. Générale en ce sens que les règles relatives à la sécurité des produits 2 , l'étiquetage et la présentation 3 , et en matière de responsabilité civile 4 sont applicables aux aliments santé comme à tout autre produit. Spécifique dans la mesure où des textes plus précis concernent plus directement et/ou exclusivement les aliments santé. En synthétisant à l'extrême les règles applicables à la matière, l'idée directrice est de tout autoriser sous réserve de respecter deux limites fondamentales : ne pas tromper le consommateur et rester en dehors du champ des médicaments. En pratique, il conviendra donc, avant même de procéder à la commercialisation d'un aliment santé, de s'interroger sur sa nature juridique - ou sa qualification - qui conditionnera la réglementation applicable au stade de sa mise sur le marché. 
Summary : One of the problems encountered when talking about "health foods" is the lack of common definition. We can consider that they include traditional foods with health claims, functional foods, fortified traditional foods, food intended for a particular nutritional use (PARNUT) and foods supplements. In European law, all products necessarily need to be defined either as foodstuff or as medicine. Consequently, practical questions must be borne in mind when marketing foodstuffs in the EU: is it a food, to which category of food does it belong, and are there any particular regulations applying to this product? According to the legal nature of the product, one should examine under which system and, if needed, under which procedure it can be marketed. The principle is that for foodstuffs in general, marketing is free, without pre-market approval or prior declaration and under the sole responsibility of the person responsible for the first marketing of the product (manufacturer or importer), only subject to a posteriori controls. However, new substances are subject to pre-market approval, according to various procedures depending on the case (EU procedure for "novel foods", French procedure under the Decret of 1912). Concerning the presentation of the product, it is also necessary to determine to which category the product belongs, because some particular labelling rules may apply. In any case, claims should not be misleading. It is also prohibited to attribute to any foodstuff the property of preventing, treating or curing a human disease, or refer to such properties. Nutrition facts are not mandatory in general, but they are (and in that case they are strictly regulated) when a nutrition claim is made. Health claims in general are presently a highly debated topic.

Keywords : health foods, drugs, functional foods, supplements foods, fortified foods, novel foods, PARTNUT, claims, labelling.

\section{ARTICLE}

\section{Nature juridique de l'aliment santé}

Le caractère hybride des aliments santé n'est pas sans soulever quelques difficultés d'appréciation. D'emblée, il convient de s'interroger sur la nature juridique du produit : s'agit-il d'une denrée alimentaire ou d'un médicament ? Si c'est une denrée alimentaire, à quelle catégorie appartient-il ?

\section{Denrée alimentaire ou médicament}

\section{La problématique générale}

Si la denrée alimentaire et le médicament ont des fonctions différentes, il n'en reste pas moins vrai qu'ils jouent, l'un et l'autre, un rôle complémentaire dans la santé du consommateur. Or, la législation interdit l'assimilation entre les deux catégories de produits. Dès lors, la question qu'il convient de se poser d'emblée est celle de savoir si le produit en cause entre dans la catégorie des denrées alimentaires ou s'il est susceptible d'être classé dans la catégorie des médicaments. Cette question est d'autant plus importante lorsque le produit considéré est un « aliment santé ». 
En effet, aucune des notions que recouvre cette désignation n'est reconnue en droit français pour désigner une catégorie de produits en tant que telle, tout produit étant nécessairement une denrée alimentaire ou un médicament.

À l'heure actuelle, il n'existe pas de définition communautaire de la denrée alimentaire. En droit français, cette dernière est définie comme " toute denrée, produit ou boisson, destiné à l'alimentation de l'homme " (article R. 112-1 du Code de la consommation), ce qui est purement descriptif et n'apporte guère d'information susceptible de simplifier la problématique. Le médicament est pour sa part défini par la directive $65 / 65 / \mathrm{CE}^{5}$ comme " une substance ou une préparation présentée comme possédant des propriétés curatives ou préventives à l'égard des maladies humaines ou animales" (médicament par présentation) ainsi que comme " tout produit pouvant être administré à l'homme ou l'animal, en vue d'établir un diagnostic médical ou de restaurer, corriger ou modifier leurs fonctions organiques " (médicament par fonction).

En pratique, le point de départ de toute analyse juridique d'un produit, a fortiori d'un aliment santé, est la définition du médicament. Est denrée alimentaire ce qui n'est pas médicament (ni par présentation, ni par fonction).

Cette démarche paraît simple a priori, mais son application est fort complexe dans la mesure où la notion de médicament doit à son tour être interprétée et, de fait, diffère selon les États membres de I'Union européenne. Elle est conçue de manière extensive par les autorités françaises qui font prévaloir une interprétation large de la notion de médicament, en particulier en matière de médicament par présentation, tandis que certains États ${ }^{6}$ se montrent plus ouverts. Plus gravement, cette situation est admise par la Cour de justice des Communautés européennes, qui considère qu'en la matière l'harmonisation n'est pas totale et que les États membres disposent d'une grande latitude pour protéger la santé de leurs citoyens.

À cet égard, les aliments dits « fonctionnels » sont sans doute les plus difficiles à cerner. On admet en général que l'aliment remplit deux rôles essentiels : nutritionnel, via l'apport énergétique et l'apport en micronutriments (tels que les vitamines et les minéraux), et sensoriel, par le goût, les odeurs, la texture, la couleur. Le concept d'aliment fonctionnel ajoute une troisième dimension, celle du maintien du bon état physiologique de l'organisme, de la préservation à long terme d'un état optimal de bonne santé, physique et psychique, qui ne se confond pas nécessairement avec la fonction nutritionnelle tout en en étant très proche.

\section{Le cas des compléments alimentaires}

Le droit français définit à l'heure actuelle le complément alimentaire comme " tout produit destiné à être ingéré en complément de l'alimentation courante, afin de pallier l'insuffisance réelle ou supposée des apports journaliers ${ }^{7}$.

Malgré son existence, il est à noter que cette définition n'entraîne pas de conséquence juridique particulière quant au régime du produit qu'elle désigne. Selon le cas, et en fonction des critères énoncés ci-dessus, les compléments alimentaires peuvent être des médicaments (par présentation) ou des denrées alimentaires (de consommation courante ou destinées à une alimentation particulière, traditionnelle ou novel food). 
Les compléments alimentaires font actuellement l'objet d'un projet d'harmonisation communautaire présenté par la Commission européenne en 2000 et actuellement en cours d'examen par le Conseil et le Parlement. Ce texte ne vise à ce stade que les vitamines et les minéraux, mais il a vocation à être étendu ultérieurement à d'autres catégories (notamment les acides aminés). Dans ce texte, les compléments alimentaires sont définis comme " les denrées alimentaires qui constituent une source concentrée de nutriments ou d'autres substances ayant une fonction nutritionnelle ou physiologique, seuls ou combinés, qui sont commercialisés sous forme de doses et dont le but est de compléter l'apport de ces nutriments dans un régime alimentaire normal ».

Il est intéressant de noter que les compléments alimentaires sont ici clairement classés dans les denrées alimentaires, et donc hors de la sphère du médicament. Toutefois, la proposition communautaire reprenant l'interdiction, commune à toutes les denrées alimentaires, des allégations relatives à la prévention des maladies, un complément alimentaire conforme quant à sa composition aux dispositions de la directive pourra bien se voir qualifier de médicament par présentation s'il porte des allégations jugées trop « médicales».

\section{Le cas particulier des plantes}

Dans le domaine des plantes, le droit français renvoie en principe au monopole de la pharmacie (article L. 4211-1 $5^{\circ}$ du Code de la santé publique) : « sont réservés aux pharmaciens (...) la vente des plantes médicinales inscrites à la pharmacopée, sous réserve des dérogations établies par décret ». Deux conditions cumulatives sont donc exigées pour qu'une plante relève du monopole de la pharmacie : son caractère de plante médicinale et son inscription à la pharmacopée.

Comme le précise une circulaire du 2 juillet 1979, toutes les plantes inscrites à la pharmacopée ne sont donc pas médicinales. En l'absence de définition donnée par la législateur, on considère comme plantes médicinales " uniquement celles qui ont des usages exclusivement médicinaux à l'exception de tout usage alimentaire, condimentaire ou hygiénique ».

En vertu du décret 79-480 du 15 juin 1979 "relatif à la vente au public des plantes médicinales ", certaines plantes considérées comme médicinales sont également "libérées " ${ }^{8}$ à condition qu'elles soient vendues " en l'état " : l'extraction sélective est donc interdite ; il est toutefois possible de fractionner ces plantes (sous forme de poudre) et de les présenter sous forme de comprimés ou de gélules. En revanche, sont interdits les mélanges, sauf pour quelques espèces ${ }^{9}$.

Une réflexion d'ensemble est actuellement en cours sous l'égide de l'Afssaps (Agence française de sécurité sanitaire des produits de santé) sur le régime des plantes, dans le but notamment de réviser la liste des plantes « libérées ».

\section{Quelle catégorie de denrée alimentaire?}

La seconde question qu'il convient de se poser en présence d'un " aliment santé " qui ne relève pas de la catégorie des médicaments est celle de savoir si l'on est en présence d'une denrée de consommation courante ou d'une denrée destinée à une alimentation particulière. 
Il convient aussi, pour les denrées de consommation courante, de vérifier s'il existe ou non un texte définissant le produit et en fixant les caractéristiques. Dans tous les cas, se pose aussi la question de savoir s'il s'agit d'un aliment « traditionnel » ou d'un « novel food ».

\section{Denrée de consommation courante ou denrée destinée à une alimentation particulière (DDAP)}

Telles que définies par la directive $89 / 398 / \mathrm{CE}^{10}$, les denrées destinées à une alimentation particulière sont « celles qui, du fait de leur composition particulière ou du procédé particulier de fabrication, se distinguent nettement des denrées de consommation courante, conviennent à l'objectif nutritionnel indiqué et sont commercialisées de manière à indiquer qu'elles répondent à cet objectif ". La directive prévoit neuf catégories spécifiques de DDAP, dont quatre ont fait l'objet de directives d'harmonisation ${ }^{11}$.

En droit français, outre la transposition des directives communautaires, on trouve plusieurs dispositions nationales relatives à des catégories de produits déterminés ${ }^{12}$.

Enfin, il ne faut pas perdre de vue que, l'alimentation particulière étant définie d'une manière générique en fonction de sa finalité et de sa présentation et non selon une liste finie de catégories de produits, il est toujours possible d'envisager des produits pour une catégorie ciblée de population non explicitement prévue par un texte ${ }^{13}$.

Une denrée alimentaire qui n'entre pas dans la catégorie des DDAP est une denrée de consommation courante.

\section{Produit réglementé ou non}

Bien que la question se pose rarement dans le domaine des " aliments santé ", il ne faut pas non plus négliger l'hypothèse où un produit serait déjà couvert par une réglementation (communautaire ou française) le décrivant, réglementant sa composition et réservant la dénomination à certaines caractéristiques. La supplémentation du produit traditionnel, ou l'ajout de substance à but nutritionnel ou fonctionnel spécifique, peut alors faire perdre à ce produit la dénomination à laquelle il aurait droit selon la recette traditionnelle ${ }^{14}$.

Si la définition du produit relève d'un texte français, il peut aussi être utile de vérifier si cette définition est conforme à la définition communautaire (quand elle existe) ou si, éventuellement, elle n'est pas constitutive d'une entrave technique aux échanges dans I'Union Européenne (si le produit provient d'un autre État membre où il est librement produit et commercialisé).

\section{Denrée traditionnelle ou « novel food »}

Les " aliments nouveaux " ${ }^{15}$ ou " novel foods » sont définis au règlement 258/97/CE instituant un régime spécifique d'autorisation de mise en marché ${ }^{16}$. Sont concernés : les produits dont la consommation est restée négligeable dans la Communauté (jusqu'en mai 1997, date d'entrée en vigueur du règlement) et qui relèvent de l'une des six catégories énoncées à l'article $1^{\text {er }} \S 2$ du règlement ${ }^{17}$.

En principe, donc, tous les produits n'entrant dans aucune de ces six catégories doivent être considérés comme produits traditionnels (produits de consommation courante ou destinés à une alimentation particulière). En pratique, c'est le premier critère qui s'impose d'emblée pour tout 
opérateur responsable de la première mise sur le marché d'un produit. II doit se demander si son produit est ou non un produit " nouveau "(au sens du règlement 258/97). Une fois encore, la réponse est déterminante puisque de celle-ci dépend le régime de mise sur le marché ${ }^{18}$.

\section{Réglementation applicable}

La multiplicité des réglementations applicables aux aliments santé ne va pas sans difficulté, leur mise en œuvre devant être assurée de façon cohérente. La réglementation actuellement applicable en France s'articule essentiellement autour de l'information et de la protection du consommateur. Elle est plus ou moins restrictive et dépend du type de produit en question. II convient donc d'être très attentif, non seulement à la composition du produit, mais aussi à sa présentation et en particulier aux allégations employées.

\section{Règles de mise sur le marché}

Les règles de mise en marché, tant de fond (composition) que de procédure dépendent directement de la nature juridique du produit telle que définie plus haut.

\section{Cas général}

Le principe est que, pour les denrées alimentaires en général, la mise à la consommation est libre, sans autorisation ni déclaration préalable, et ce sous la seule responsabilité du responsable de la première mise en marché en France (fabricant ou, selon le cas, importateur). Les éventuelles infractions à la réglementation relèvent donc de seuls contrôles a posteriori en vertu de l'obligation générale de conformité et de sécurité des produits ${ }^{19}$.

Néanmoins, et indépendamment du cas de l'alimentation particulière et des "novel food » (voir ciaprès), les denrées dans lesquelles sont incorporées des substances qui n'ont encore jamais été autorisées en France doivent faire l'objet d'une autorisation préalable sur base d'un dossier scientifique soumis à l'Afssa (Agence française de sécurité sanitaire des aliments).

En effet, en vertu du décret du 15 avril 1912, toujours en vigueur, il est interdit " de détenir en vue de la vente, de mettre en vente ou de vendre toutes marchandises et denrées destinées à l'alimentation humaine lorsqu'elles ont été additionnées de produits chimiques autres que ceux dont l'emploi est déclaré licite par les arrêtés pris de concert par le ministre de l'Agriculture et du Développement rural, le ministre de l'Économie et des Finances, le ministre du Développement industriel et scientifique et le ministre de la Santé publique, sur l'avis de I'Agence française de sécurité sanitaire des aliments (Afssa)».

Par ailleurs, s'agissant des produits n'ayant pas le statut de médicament mais présentés comme étant " bénéfiques pour la santé ", une procédure spécifique au droit français doit être respectée en matière d'étiquetage et de présentation. ॥ s'agit du visa de publicité (« visa pp »), sur lequel on reviendra ultérieurement dans le cadre de l'examen des règles de présentation des produits. 


\section{Les règles spécifiques à certaines catégories de produits}

\section{* L'alimentation particulière}

Lorsque le produit considéré relève de la catégorie des DDAP, sa mise sur le marché est soumise à un régime particulier en application de la réglementation communautaire ; la situation française reste toutefois assez complexe :

- s'il s'agit d'un produit appartenant à un groupe ayant déjà fait l'objet d'une réglementation spécifique (communautaire ou nationale), la mise sur le marché ne requiert aucune formalité particulière et son régime est analogue à celui des denrées de consommation courante (sous réserve évidemment du respect des prescriptions de fond propres à chaque produit) ;

- si le produit n'appartient pas à un groupe ayant fait l'objet de règles spécifiques, plusieurs hypothèses peuvent de présenter :

- s'il contient une substance non encore mise sur le marché en France, ou si une substance déjà autorisée est employée dans des conditions non encore admises ${ }^{20}$, l'obligation de soumission à une procédure d'autorisation préalable examinée ci-dessus s'applique ;

- si le produit ne contient pas de telles substances, il est en principe librement mis sur le marché mais doit faire l'objet d'une déclaration (accompagnée d'un exemplaire de l'étiquetage) auprès du préfet du département où a lieu la fabrication ou l'importation. Si le produit a déjà été mis en vente dans un autre État membre de I'Union européenne, la déclaration doit également être accompagnée de l'indication de l'autorité de l'État membre destinataire de la première déclaration. En outre un dossier scientifique permettant de justifier de la conformité du produit aux dispositions réglementaires et de la véracité des allégations qui l'accompagnent doit être tenu à disposition de l'Administration en cas de contrôle (contrôle a posteriori).

* Les novel foods

La mise sur le marché de ces produits est régie par le Rt. CE 258/97, précité. Contrairement aux aliments traditionnels, les nouveaux aliments sont soumis à l'obtention d'une autorisation de mise sur le marché ou, si le produit est reconnu « substantiellement équivalent » à un aliment traditionnel, à une simple notification.

L'autorisation est une procédure communautaire complexe, initiée dans l'État membre de première mise en marché mais qui fait intervenir les autres États membres et qui est conduite sous l'égide la Commission européenne. Cette procédure d'autorisation concerne à la fois le produit, sa composition et sa présentation.

\section{Les règles de présentation : les allégations}

La réglementation applicable en la matière repose sur deux exigences de base : ne pas induire le consommateur en erreur et, si l'on souhaite rester dans la catégorie des denrées alimentaires, ne pas empiéter sur le domaine du médicament. La directive communautaire relative à l'étiquetage ${ }^{21}$ prévoit expressément qu'il est « interdit de faire état de propriétés de prévention, de traitement et de guérison d'une maladie humaine ou d'évoquer ces propriétés ${ }^{22}$. 
Rappelons aussi que, outre la nature du produit, sa présentation est également déterminante pour sa classification. Un même produit, en fonction de la communication faite à son sujet, relèvera ou non, non seulement du médicament par présentation ou de l'aliment, mais éventuellement de la catégorie des denrées destinées à une alimentation particulière ou de celle de l'alimentation courante, selon que l'on mette en avant ou non un certain objectif nutritionnel. Selon la stratégie choisie, il conviendra donc de mettre en avant des qualités pouvant faire entrer un produit dans la catégorie des DDAP ou, au contraire, prendre soin de s'en abstenir si l'on ne souhaite pas le soumettre au régime particulier régissant cette catégorie de produits.

L'étiquetage et la présentation sont soumis à de nombreuses règles dont l'objectif premier est la protection et l'information du consommateur. Dans le cadre du présent article, nous nous concentrons sur les règles relatives aux allégations, étant précisé que, bien entendu, toutes les autres règles sont également à prendre en compte.

Bien qu'encore réglementées de manière très parcellaire, sinon pour être soumises aux règles générales d'interdiction des tromperies, les allégations ${ }^{23}$ relatives aux " aliments santé ", tels que définis de manière large en introduction du présent article, peuvent se définir comme celles qui indiquent, suggèrent ou impliquent qu'une relation existe entre un aliment, un élément nutritif ou une substance contenue dans l'aliment et un état lié à la santé ou une modification d'un paramètre biologique, mais sans faire référence à la maladie ${ }^{24}$.

Aux fins du présent article, et sur la base de différents écrits épars, il paraît possible de distinguer les catégories d'allégations suivantes :

- allégations nutritionnelles ;

- allégations fonctionnelles ;

- allégations « santé ";

- allégations de « réduction de risque »;

- allégations médicales.

\section{Les allégations nutritionnelles}

Telles que définies par le décret 93/1130 du 27 septembre $1993^{25}$, les allégations nutritionnelles consistent " en toute représentation et tout message publicitaire qui énonce, suggère ou indique qu'une denrée alimentaire possède des propriétés nutritionnelles particulières, soit en raison de l'énergie (valeur calorique) qu'elle fournit ou ne fournit pas, ou qu'elle fournit à un taux réduit ou accru, soit en raison des nutriments qu'elle contient ou ne contient pas, ou qu'elle contient en proportion réduite ou accrue $»^{26}$.

Les allégations nutritionnelles ainsi définies sont libres, mais leur présence déclenche l'obligation d'étiquetage nutritionnel ${ }^{27}$, c'est-à-dire l'indication de la teneur en énergie et en nutriment pour 100 $\mathrm{g}$ du produit. Selon les allégations qui sont faites, cet étiquetage doit comporter des mentions obligatoires dans des conditions précises. 
En toute hypothèse, l'étiquetage nutritionnel minimum comporte l'indication de la valeur calorique, des glucides, des lipides et des protéines.

D'autres indications s'ajoutent en fonction du choix de l'opérateur et/ou des allégations figurant sur le produit ${ }^{28}$.

S'agissant de l'indication de vitamines ou de minéraux dans l'étiquetage nutritionnel, il faut tenir compte en même temps des règles françaises complexes régissant la restauration, autorisée dans certaines conditions pour les denrées de consommation courante ${ }^{29}$, et la supplémentation qui est, en principe, réservée aux DDAP ${ }^{30}$.

\section{Les allégations fonctionnelles}

Les allégations fonctionnelles peuvent se définir comme celles qui ont " pour objet d'informer le consommateur sur le rôle physiologique d'un nutriment ou d'une substance contenu dans une denrée alimentaire $"{ }^{31}$. Selon ce même avis de la Cedap, les allégations fonctionnelles ne sont possibles que lorsque les éléments faisant l'objet de l'allégation disposent d'un apport quotidien recommandé (AJR) ou d'un "rôle fondé scientifiquement et admis dans les recommandations diététiques généralement reconnues comme scientifiquement établies ". En outre, toujours selon la Cedap, de telles allégations doivent être interdites si elles :

- ne peuvent être justifiées ${ }^{32}$;

- sont susceptibles de dénigrer l'alimentation ou d'autres denrées alimentaires, analogues ou non, par comparaison ;

- peuvent faire naître des doutes sur la sécurité d'aliments analogues, susciter la crainte ou exploiter ce sentiment chez le consommateur ;

- laissent entendre qu'une alimentation équilibrée normale ne peut fournir tous les éléments nutritifs en quantité suffisante.

Enfin, pour pouvoir porter une allégation fonctionnelle relative à une substance particulière, une denrée doit être considérée comme une source significative de l'élément nutritif en question.

\section{Les allégations " santé »}

Les allégations " santé » telles que nous les définissons ici indiquent, suggèrent ou impliquent qu'une relation existe entre un aliment, un élément nutritif ou une substance contenue dans un aliment et un état lié à la santé ou une modification d'un paramètre biologique, mais sans faire référence à la maladie ${ }^{33}$. La problématique des allégations santé est délicate dans la mesure où prévaut une définition très extensive du médicament (voir plus haut). II en résulte que si, en pratique, le consommateur paraît suffisamment protégé contre les tromperies, force est de reconnaître que la marge de manœuvre des industriels est ici extrêmement étroite. 
D'une manière générale, on peut considérer que des allégations " positives » et assez vagues faisant référence à la forme ou au bien-être sont admises, tandis que des allégations visant une maladie ou un état pathologique ne le sont pas.

Des allégations positives plus précises, généralement admises à l'heure actuelle par la DGCCRF, peuvent aussi être considérées par le ministère de la Santé et l'Afssaps comme relevant du visa de publicité (visa p.p.).

II s'agit là d'une autorisation préalable, prévue à l'article L-5122.14 du Code de la santé publique, qui concerne au terme de cet article " les produits autres que les médicaments mais présentés comme favorisant la prévention ou le traitement de maladies, ou encore la restauration ou la modification de fonctions organiques ". Relèvent donc en principe de ce régime toute une série de denrées alimentaires comportant des "allégations santé ", qui sont ainsi soumises à une procédure particulière d'étiquetage sans pour autant relever du régime du médicament. Le ministère de la Santé précise bien que ce visa n'est accordé que si le produit ne relève pas de la réglementation du médicament, ni par sa présentation (notamment des allégations thérapeutiques à l'égard des maladies), ni par sa fonction (en raison de ses propriétés effectives) et, bien entendu, si les propriétés bénéfiques alléguées sont reconnues exactes ${ }^{34}$.

Il convient néanmoins de savoir que la question du visa p.p., procédure typiquement française, est actuellement controversée. On peut en effet se demander comment il est possible, au regard des règles d'étiquetage des denrées alimentaires qui relèvent du droit communautaire, d'instituer une procédure d'autorisation préalable au terme de laquelle, qui plus est, seraient autorisées des allégations allant plus loin que ce qui est normalement autorisé pour les denrées alimentaires.

Ceci explique que certains industriels ressentent cette obligation de visa comme une contrainte inutile, tandis que d'autres peuvent au contraire considérer qu'elle leur offre une possibilité d'allégations fortes qui ne seraient pas autorisées autrement.

\section{Les allégations de "réduction de risque "}

Entrent dans cette catégorie des allégations qui, sans viser directement la prévention d'une maladie

${ }^{35}$, font état de la contribution du produit à la diminution des risques ${ }^{36}$. À une époque où les risques de maladies chroniques (dues notamment à l'allongement de la durée de vie) sont de plus en plus importants par rapport aux risques de maladies aiguës, le besoin et, donc, le marché de produits pouvant faire état de telles propriétés sont considérables.

Le débat sur ce terrain est actuellement ouvert, l'industrie demandant la possibilité de faire de telles allégations tandis que la plupart des pouvoirs publics (notamment les autorités françaises et la Commission européenne) s'y montrent dans l'ensemble opposés ${ }^{37}$. Nul doute que l'actualité en ce domaine méritera d'être suivie de très près dans les mois à venir.

\footnotetext{
* Les allégations « médicales »
} 
Il s'agit là d'allégations entrant clairement dans le domaine du médicament, comme exposé précédemment ${ }^{38}$. Conceptuellement, le sort de telles allégations ne fait guère de doute : elles sont interdites pour les denrées alimentaires, et donc pour ce que nous avons désigné sous le terme général d'« aliment santé ».

Il reste à déterminer, dans chaque cas, si on est en présence d'allégations " médicales ", ce qu'il n'est pas toujours facile d'évaluer. Outre le cas, encore relativement net, des allégations de réduction de risque que l'on vient d'évoquer, celui des allégations soumises en France au visa p.p. nous paraît aussi ajouter à la confusion. En effet, comment peut-on dire qu'un produit « présenté comme favorisant la prévention ou le traitement de maladies » n'est pas un « médicament par présentation » ?

Assurément, en France du moins, des clarifications s'imposent pour assurer la sécurité juridique des opérateurs. De telles clarifications devraient, et cela va de soi, être en conformité avec le droit communautaire - actuel ou en formation.

Les clarifications sont d'autant plus nécessaires que, bien entendu, la violation des règles que l'on vient d'exposer peut entraîner des conséquences - civiles ou pénales - non négligeables.

\section{Les sanctions encourues en cas de manquements à la réglementation applicable}

Plusieurs types d'infractions peuvent être commises par le fabricant ou l'importateur d'un aliment santé. Les plus fréquentes consistent en une erreur sur la qualification juridique du produit, entraînant une violation de l'obligation générale de conformité et le non respect des obligations en matière d'étiquetage et de présentation.

* Erreurs de qualification du produit

Au stade de la mise sur le marché, plusieurs erreurs de qualification sont possibles, les plus fréquentes consistant à commercialiser comme denrée alimentaire un produit qui sera considéré comme un médicament, ou à se positionner sur une catégorie erronée de denrées alimentaires.

Dans de tels cas, les risques sont alors multiples, pouvant provenir de poursuites de l'Administration ou de réactions de consommateurs et/ou de concurrents :

- risque pénal : tromperie, falsification, voire exercice illégal de la pharmacie, sans compter les risques fiscaux car la TVA en matière de médicament et de denrée alimentaire n'est pas la même ;

- risque civil : demande de dommages et intérêts de consommateurs (individuels ou via des associations) s'estimant trompés, ou d'autres opérateurs s'estimant victimes de concurrence déloyale.

* Manquements à l'obligation générale de conformité et de sécurité 
Indépendamment des infractions spécifiques ci-dessus évoquées, une obligation générale de conformité et de sécurité des produits, inscrite dans le Code de la consommation (article L. 212-1) pèse sur le responsable de la première mise sur le marché, c'est-à-dire selon le cas le fabricant en France ou l'importateur. C'est donc ce dernier qui est responsable de la conformité des produits et qui fera l'objet, le cas échéant, de poursuites.

L'obligation générale de sécurité implique le risque d'être poursuivi pour mise en marché d'un produit non autorisé présentant un risque pour la santé. Dans ce cas, et si les tribunaux reconnaissent ce risque réellement fondé, il pourra être difficile, en l'état actuel de la jurisprudence et du droit communautaire, de faire déclarer la réglementation française contraire au droit communautaire et de se prévaloir de la libre circulation des marchandises dans l'Union européenne.

L'enjeu d'erreurs dans l'interprétation et l'application de la réglementation peut donc être, on le voit, d'importance.

\section{Notes:}

${ }^{1}$ Nous écartons délibérément tout emploi de termes tels que " alicament », " nutraceutique », etc.

${ }^{2}$ Article 212-1 du Code de la consommation.

${ }^{3}$ Notamment la directive 2000/13 du 20 mars 2000 sur l'étiquetage et la présentation des denrées alimentaires (transposée en droit français dans le Code de la consommation).

${ }^{4}$ Notamment l'article $1386-1$ et suivants du Code civil en matière de responsabilité des produits défectueux.

${ }^{5}$ Dont s'inspire largement l'article L. 5111-1 du Code de la santé publique, qui définit le médicament comme " toute présentation ou composition présentée comme possédant des propriétés curatives ou préventives à l'égard des maladies humaines ou animales " et comme " toute substance ou composition pouvant être administrée à l'homme ou à l'animal en vue d'établir un diagnostic médical ou de restaurer, corriger ou modifier des fonctions organiques chez l'homme ou l'animal ».

${ }^{6}$ Royaume-Uni ou Belgique par exemple.

${ }^{7}$ Décret du 15 avril 1912. Art. 15-2 modifié par le décret 97-964 du 14 octobre 1997.

${ }^{8}$ C'est-à-dire hors du monopole de la pharmacie. Ainsi - notamment - la camomille, la gentiane, le tilleul, la verveine, la bruyère, etc. (décret du 15 juin 1979 relatif à la vente au public des plantes médicinales inscrites à la pharmacopée).

${ }^{9}$ Tilleul, verveine, camomille, menthe, oranger, cynorrhodon, hibiscus.

${ }^{10}$ Transposée en droit français par le décret 91/827 du 29 août 1991. 
${ }^{11}$ Le secteur de l'alimentation infantile, les substituts de repas pour les régimes de perte de poids et les denrées d'alimentation médicale spéciale. Les autres catégories, non harmonisées, sont les aliments pour sportifs, les aliments pour diabétiques, les aliments pour régime hyposodé et les aliments sans gluten.

${ }^{12}$ Arrêté du 20 juillet 1997 sur les produits diététiques et de régime (produits de régime d'apport protidique ou d'apport lipidique particulier, divers produits à teneurs garanties).

${ }^{13}$ Cette possibilité peut se révéler d'autant plus intéressante que, en droit français du moins, l'enrichissement en vitamines et minéraux n'est autorisé que pour l'alimentation particulière, et n'est pas possible pour l'alimentation de consommation courante.

${ }^{14}$ Par exemple, les jus de fruit et le chocolat sont définis dans des directives communautaires. La plupart des produits laitiers sont définis par textes communautaires ou, selon le cas, français.

${ }^{15}$ II ne faut pas confondre ici les « aliments nouveaux » au sens de " novel foods » et le qualificatif « nouveau » qui peut figurer sur l'étiquetage et/ou l'emballage de certains produits.

${ }^{16}$ Certains produits sont exclus du champ d'application du règlement 258/97/CE : les additifs, les arômes et les solvants d'extraction qui font l'objet de directive spécifiques.

${ }^{17}$ À savoir : - « les aliments et ingrédients alimentaires contenant des organismes génétiquement modifiés au sens de la directive 90/220/CE ou consistant en tels organismes ;

- les aliments et ingrédients alimentaires produits à partir d'organismes génétiquement modifiés mais n'en contenant pas ;

- les aliments et ingrédients alimentaires présentant une structure moléculaire primaire nouvelle ou délibérément modifiée ;

- les aliments et ingrédients alimentaires composés de micro-organismes, de champignons ou d'algues ou isolés à partir de ceux-ci ; - les aliments et ingrédients alimentaires composés de végétaux ou isolés à partir de ceux-ci et les ingrédients alimentaires isolés à partir d'animaux, à l'exception des aliments et des ingrédients alimentaires obtenus par des pratiques de multiplication ou de reproduction traditionnelles et dont les antécédents sont sûrs en ce qui concerne l'utilisation en tant que denrées alimentaires;

- les aliments et ingrédients alimentaires auxquels a été appliqué un procédé de production qui n'est pas couramment utilisé lorsque ce procédé entraîne dans la composition ou dans la structure des aliments ou des ingrédients alimentaires des modifications significatives de leur valeur nutritive, de leur métabolisme ou de leur teneur en substances indésirables ».

${ }^{18}$ La réponse n'étant pas toujours évidente, le règlement a prévu la possibilité de faire intervenir le comité permanent des denrées alimentaires pour déterminer si un type d'aliment ou d'ingrédient alimentaire relève d'une des catégories couvertes par le texte.

${ }^{19}$ Articles L. 212-1 et L. 212-1 du Code de la consommation. 
${ }^{20}$ Par exemple, supplémentation en vitamines en quantité différente de celle précédemment autorisée, ou selon une combinaison nouvelle.

${ }^{21}$ Transposée en droit français à l'article R 112-7 du Code de la consommation.

${ }^{22}$ Interdiction qui ne fait que refléter la définition du médicament par présentation telle qu'exposée plus haut.

${ }^{23}$ Directive étiquetage.

${ }^{24}$ II n'existe pas actuellement de texte communautaire sur les allégations. On notera toutefois un document de travail de la Commission en date du 29 mai 2001 sur les allégations nutritionnelles et les allégations fonctionnelles. En droit français, parmi les textes de référence, on citera l'avis du CNA (Conseil national de l'alimentation) du 30 juin 1998 sur les allégations faisant un lien avec la santé et I'avis de la CEDAP du 18 décembre 1996 sur les allégations nutritionnelles fonctionnelles.

${ }^{25}$ Pris pour la transposition de la directive 90/496 du 24 septembre 1990.

${ }^{26}$ Par exemple : « riche en calcium ».

27 Inversement, l'étiquetage nutritionnel n'est pas obligatoire en l'absence d'allégations nutritionnelles, mais il peut néanmoins être effectué dans les conditions prévues au décret.

${ }^{28}$ Voir le détail des obligations au décret 93/1130.

${ }^{29}$ La restauration, consistant à réintroduire des vitamines ou minéraux pour compenser la perte de ces nutriments en cours de fabrication du produit, permet l'allégation « à teneur garantie en... ».

${ }^{30}$ La supplémentation, dans la mesure où elle est autorisée, permet l'indication " source de... " ou " riche en... ". II n'entre pas dans le cadre de cet article de développer les conditions, très complexes, dans lesquelles ces allégations sont possibles selon le cas. Il faudra toujours se reporter à l'ensemble de la réglementation régissant le produit considéré.

${ }^{31}$ Avis de la Cedap (à présent remplacée par l'Afssa) du 18 décembre 1996. Par exemple : « influence du calcium sur les os $"$.

${ }^{32}$ Un avis relatif au caractère trompeur des allégations nutritionnelles a été rendu par la Cedap en octobre 1997. Il fait état des revendications considérées comme établies sur le plan scientifique. Pour toute autre allégation, des justificatifs scientifiques restent nécessaires au cas par cas.

${ }^{33}$ Par exemple : " le calcium améliore la densité osseuse » (et non " prévient l'ostéoporose », ce qui serait une allégation " médicale » interdite pour une denrée alimentaire). Ou encore : "le jus de fruits (qui contient... calcium) aide à maintenir le capital osseux ».

${ }^{34}$ On peut se référer aux lignes directrices émises en 1993 par le ministère de la Santé, qui n'ont pas encore été actualisées à ce jour et doivent donc être considérées comme toujours valables.

${ }^{35}$ Comme serait l'allégation : « Le calcium (ou ce jus de fruits) prévient l'ostéoporose. »

${ }^{36}$ " Le calcium (ou ce jus de fruits) contribue à diminuer les risques d'ostéoporose. » 
37 Beaucoup considèrent en effet que de telles allégations sont en réalité des allégations de prévention de maladie, c'est-à-dire des allégations réservées au médicament.

${ }^{38}$ " Le calcium (ou ce jus de fruit) prévient l'ostéoporose. »

\section{CONCLUSION}

L'aliment n'est pas un médicament, même lorsqu'il fait état de propriétés relatives à la santé.

On l'a vu, la libre circulation des aliments santé dans I'Union européenne n'est pas réellement assurée, puisque l'harmonisation n'est pas complète et que les États peuvent exciper de la protection de la santé publique. Néanmoins, il appartient toujours à l'État membre de prouver la nocivité ou le caractère trompeur d'un produit importé. De plus, certaines procédures d'autorisation sont désormais européennes (novel foods et, bientôt, compléments alimentaires à base de vitamines et de minéraux). Et même dans les cas où la législation nationale reste applicable (dans les matières non harmonisées), la pression des importateurs est un facteur d'évolution, à terme, incontournable.

II ressort de ce qui précède que le classement d'un produit dans une catégorie ou l'autre détermine son régime juridique, et donc son mode de commercialisation. II convient donc de l'envisager dès le début de la conception d'un produit.

La mise sur le marché d'un produit doit donc être le fruit d'une véritable stratégie, non seulement commerciale, mais aussi et tout autant juridique. En pratique, l'opérateur ne devra pas nécessairement partir de la nature de son produit ou de la catégorie juridique à laquelle il est susceptible d'appartenir comme s'il s'agissait d'une donnée intangible. Compte tenu de tous les impératifs que nous avons évoqués, il lui sera nécessaire, dans bien des cas, de déterminer en premier lieu, ou en tout cas en même temps que la conception du produit, comment il veut se positionner sur le marché, c'est-à-dire quelle communication il entend développer.

Autrement dit, il devra se demander quelles allégations, quel étiquetage et quelle publicité il souhaite privilégier; c'est en fonction de cette présentation que se fera alors le choix de catégorie juridique (DDAP, denrée de consommation courante bénéficiant ou non d'un étiquetage nutritionnel, novel food, etc.) De fait, la présence de tel ou tel ingrédient dans le produit ou l'emploi de tel ou tel procédé pourra se révéler déterminant pour ouvrir - ou fermer - les possibilités juridiques quant au choix de telle ou telle catégorie. Dès lors on ne saurait trop insister sur l'importance qu'il y a de définir globalement les orientations le plus tôt possible dans le processus de conception et de fabrication du produit, en fonction du marché ciblé. 